R E S E $\tilde{N}$ A S

\title{
¿Cómo aprenden los docentes? Tránsitos entre cartografías, experiencias, corporeidades y afectos
}

\author{
Paula Estalayo Bielsa*
}

Recibido: 24 de julio de 2020 Aceptado: 13 de agosto de 2020 Publicado: 30 de septiembre de 2020

To cite this article: Estalayo Bielsa, P. (2020). Reseña de Hernández y Hernández, F., Aberasturi Apraiz, E., Sancho Gil, J.M. y Correa Gorospe, J.M. (eds.) (2020) ¿Cómo aprenden los docentes? Tránsitos entre cartografías, experiencias, corporeidades y afectos. Barcelona: Octaedro. Márgenes, Revista de Educación de la Universidad de Málaga, 1 (3), 387-389

DOI: https://dx.10.24310/mgnmar.v1i3.10012

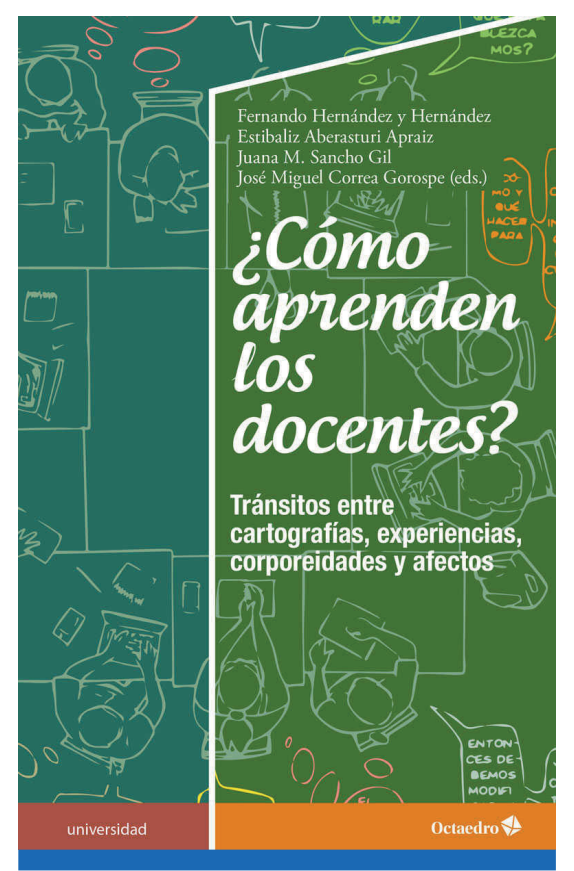

Cómo aprenden los docentes? Tránsitos entre cartografías, experiencias, corporeidades y afectos (Hernández y Hernández, F., Aberasturi Apraiz, E., Sancho Gil, J.M. y José Miguel Correa Gorospe, J.M. (eds.) 2020, Barcelona. Ediciones Octaedro. 280 páginas ISBN: 978-84-17667-91-7
Una cuestión tan fundamental como es conocer el proceso de aprendizaje de los docentes ha sido, paradójicamente, poco explorado en los estudios sobre el profesorado. Incluso dentro de la formación docente, tanto de Infantil, como de Primaria y Secundaria, no son comunes los espacios ni las oportunidades para trabajar dicha cuestión. Este es el foco principal de “¿Cómo aprenden los docentes? Tránsitos entre cartografías, experiencias, corporeidades y afectos”. El libro supone una contribución a un tema clave como es comprender el aprendizaje. Muestra que "el modo como el profesorado se concibe a sí mismo como aprendiz afecta a su concepción y a su manera de trabajar con los estudiantes" (p. 123).

El libro emerge de la investigación APREN-DO (EDU201570912-C2-1-R), un proyecto llevado a cabo por los grupos de investigación Esbrina y Elkarrikertuz. Ambos tienen el objetivo de que su actividad investigadora "vuelva a los escenarios de los que parte y para quienes se realiza la investigación educativa: los docentes y la Escuela” (p. 203). Esto no sólo queda materializado en el planteamiento de APREN-DO y las acciones que se llevaron a cabo, como se puede observar a lo largo de los capítulos; sino también en la elección de un libro como formato de escritura para dar cuenta de una investigación, pues permite incorporar a una audiencia más amplia que la académica, y resulta un ejercicio de transparencia y honestidad, ya que se hacen visibles dudas, tensio- 
nes, cambios y sorpresas, mostrando la inestabilidad y vulnerabilidad de los investigadores. El libro invita al lector a conocer lo que normalmente queda oculto de la investigación, sus entresijos, movimientos y la no-linealidad, es decir, el devenir del proyecto APREN-DO. Esta decisión de mostrar el proceso y no solo el resultado, hace partícipe al lector, quien puede pensar con los autores y generar nuevas preguntas.

Está estructurado en tres partes. La primera, "Sobre los fundamentos y los movimientos imprevistos” da cuenta de los desplazamientos que tuvieron lugar al principio de la investigación hacia otros marcos y otros posicionamientos como investigadores, quienes cuestionaron sus propias concepciones ontoepistemológicas y metodológicas y revisaron los fundamentos que guiaban su labor investigativa. La perspectiva poscualitativa los llevó a entender la ontología, epistemología, metodología y ética como un enredo y posibilitó que emergieran nuevos sentidos de investigar. Los autores se situaron en una investigación como proceso de inmanencia (Deleuze y Guattari, 2004) en la que no se buscan resultados concluyentes, sino que se trata de generar conceptos y trabajar con ellos; se asume así una posición de no-saber y de apertura, tomando riesgos, dejándose sorprender por lo que acontece y dialogando con ello. Además, emergieron también nuevos modos de abordar la relación, planteándose la investigación como escucha, como intercambio, como ir al encuentro del otro.

La segunda parte se llama "Cartografiar los trayectos de aprendizaje como modo de tejer relaciones entre experiencias, conocimientos y saberes”. Las cartografías fueron el sustento metodológico de la investigación, considerándolas como una metodología y una epistemología, como un instrumento de análisis, pero también de construcción de realidades. Los investigadores comenzaron haciendo sus propias cartografías de cómo aprendían, realizando aquello que después pedían al profesorado. En los capítulos se comparte lo que significó la experiencia cartográfica para investigadores y docentes. Para muchos supuso salir de su zona de confort, pues la utilización de métodos artísticos y visuales no formaba parte de sus prácticas habituales. Sin embargo, lo importante de las cartografías no era el resultado estético sino aquello que permitía pensar: éstas se conformaron como un dispositivo vivo para reflexionar sobre "la manera cómo se articulaban las diferentes capas que pueblan (...) el aprendizaje de los docentes” (p. 65). Así, los encuentros con docentes se configuraron como espacios en los que los investigadores encarnaron un rol de acompañantes, construyendo una investigación desde la reciprocidad, ya que tanto docentes como investigadores se vieron afectados por la experiencia. De esta manera, investigar con los y las docentes y no sobre ellos y ellas es uno de los ejes del proyecto APREN-DO.

"Aportaciones y derivas sobre el proceso de investigar, los afectos, el saber pedagógico y la formación docente" es la tercera y última parte, en la que se profundiza en los fundamentos teóricos que sustentaron el proyecto de investigación. La articulación de estos capítulos puede servir a docentes e investigadores a repensar teorías y prácticas pedagógicas, metodológicas e investigativas. Además, ayuda a reducir la distancia entre la investigación y la práctica educativa. Los principales ejes que se desarrollan giran en torno a la aproximación narrativa; las derivas poscualitativas; las cartografías; la noción de corporeidad; y el afecto y la afectividad, aproximándose a lo que podríamos denominar una pedagogía afectiva (Hickey Moody, 2013; Revelles Benavente, 2017).

Son muchas las aportaciones que se van dando sobre el aprender a lo largo del libro. Algunos de los hilos transversales son la revisión de la subjetividad docente desde la idea de que "el co- 
nocimiento profesional de los docentes no puede desvincularse del conocimiento de sí mismo (self), de sus experiencias biográficas, culturales, sociales, tecnológicas y afectivas” (p. 35). Así, el aprender siempre tiene lugar desde una trama de relaciones biográficas, intrapersonales y cognitivas. También tiene lugar de manera corporeizada y afectiva. Otra noción clave es la de aprender en movimiento, aprendizaje nómada (Fendler, 2013; Braidotti, 2006, 2014), que tiene que ver con desplazarse, con cambiar de lugar. El aprendizaje además tiene lugar de manera rizomática (Deleuze y Guattari, 2004), en un proceso no-lineal que nunca acaba, que no solo tiene que ver con saber algo, sino con “devenir alguien” (Radford, 2008, p. 215).

Diversas son las voces de los autores y autoras, lo que otorga al libro una gran riqueza. Diversas y valiosas son también las contribuciones que ofrece, tanto en el plano teórico, como en el metodológico. En un presente de políticas educativas neoliberales "la profesión docente nunca ha estado tan necesitada de arrojar la duda sobre su propia tarea y el alcance de sus acciones” (Correa, Aberasturi y Gutiérrez-Cabello, 2020, p. 237). Por este motivo el proyecto APREN-DO y el libro que da cuenta de él contribuyen a expandir la escucha, tejer redes y romper con el individualismo que a menudo impera en la universidad y en el mundo educativo.

\section{REFERENCIAS}

Braidotti, R. (2006). Transpositions. On Nomadic Ethics. Cambridge: Polity Press.

Deleuze, G.; Guattari, D. (2004). Mil mesetas. Capitalismo y esquizofrenia [trad. Vázquez, J.]. Valencia: PreTextos.

Fendler, R. (2013). "Becoming-Learner: Coordinates for Mapping the Space and Subject of Nomadic Pedagogy”. Qualitative Inquiry, 19(10), pp. 786-793.

Hickey Moody, A. (2013). “Affect as Method: Feelings, Aesthetics and Affective Pedagogy”. En: Coleman, R.; Ringrose, J. (eds.). Deleuze and Research Methodologies (pp. 79-95). Edimburgo: Edinburgh University Press.

Radford, L. (2008). “The ethics of being and knowing: Towards a cultural theory of learning”. En: Radford, L.; Schubring, G.; Seeger, F. (eds.). Semiotics in mathematics education: Epistemology, history, classroom and culture (pp. 215-234). Róterdam: Sense.

Revelles Benavente, B. (2017). "Affecting feminist pedagogies: performing critical thinking in between social networking sites and contemporary literature”. En: Just, E.; Wraggen, W. (eds.). Adventurous Encounters (pp. 131-55). Cambridge Scholars University Press. 